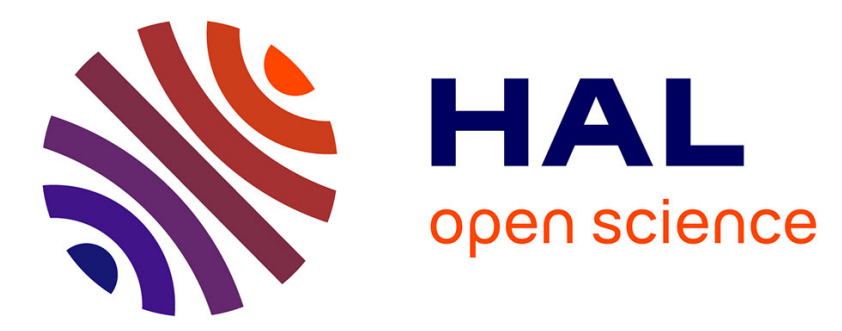

\title{
Tolérance du dispositif intra-utérin au cuivre chez les patientes nullipares : étude prospective unicentrique
}

C. Guicheteau, L. Boyer, D. A. Somé, J. Leveque, P. Poulain, M. Denier, Vincent Lavoué

\section{- To cite this version:}

C. Guicheteau, L. Boyer, D. A. Somé, J. Leveque, P. Poulain, et al.. Tolérance du dispositif intra-utérin au cuivre chez les patientes nullipares: étude prospective unicentrique. Gynécologie Obstétrique \& Fertilité, 2015, 43 (2), pp.144-150. 10.1016/j.gyobfe.2015.01.003 . hal-01147373

HAL Id: hal-01147373

https://hal-univ-rennes1.archives-ouvertes.fr/hal-01147373

Submitted on 22 May 2015

HAL is a multi-disciplinary open access archive for the deposit and dissemination of scientific research documents, whether they are published or not. The documents may come from teaching and research institutions in France or abroad, or from public or private research centers.
L'archive ouverte pluridisciplinaire $\mathbf{H A L}$, est destinée au dépôt et à la diffusion de documents scientifiques de niveau recherche, publiés ou non, émanant des établissements d'enseignement et de recherche français ou étrangers, des laboratoires publics ou privés. 
Tolérance du dispositif intra-utérin au cuivre chez les patientes nullipares : étude prospective unicentrique

Tolerance of copper intrauterine device in nulliparous patients: A single-center prospective study

C. Guicheteau a, b, L. Boyer b, c, D.A. Somé c, J. Levêque a, c, d, P. Poulain a, c, M. Denier b, V. Lavoué a, c, d

a Faculté de médecine de Rennes, université de Rennes 1, 2, Henri-Guilloux, 35000 Rennes, France

b Planning familial, 35, boulevard Delattre-de-Tassigny, 35000 Rennes, France

c Service de gynécologie obstétrique, Hôpital Sud, CHU de Rennes, 16, boulevard de Bulgarie, 35000 Rennes, France

d Inserm, ER440, OSS, CRLCC Eugène Marquis, avenue de la Bataille-FlandresDunkerques, 35000 Rennes, France

\section{Résumé}

Introduction : cette étude a pour objectif d'étudier le taux de continuation du dispositif intra-utérin (DIU) au cuivre ainsi que sa tolérance dans une population de patientes nullipares.

Matériel et méthodes : étude prospective dans un centre de planification familiale français portant sur les patientes nullipares désireuses d'une contraception par DIU au cuivre du $1^{\text {er }}$ janvier au 31 décembre 2012.

Résultats : Quatre-vingt-trois patientes ont bénéficié de la pose d'un DIU au cuivre, 11 patientes ont été perdues de vue et 72 patientes nullipares ont été inclues dans l'étude. Le 
taux de continuation de la contraception par DIU au cuivre à 1 an étaient de $90 \%$ (65/72). Le taux de satisfaction des patientes vis à vis du DIU était de $93 \%$ à 1 an. Une augmentation de l'abondance des règles est observée chez $84 \%(55 / 65)$ des patientes dont $75 \%$ (41/55) se disent peu ou pas gênées par ce symptôme. Une augmentation des dysménorrhées est observée chez 80\% (52/65) des patientes dont 58\% (30/52) se disent peu ou pas gênées par ce symptôme.

Conclusion : Le taux de continuation du DIU au cuivre à 1 an est élevé (90\%) dans la population de patientes nullipares. La tolérance est également excellente pour les patientes qui ont pu être évaluées.

Mots clés : Dispositif Intra-Utérin ; cuivre ; Nullipare 


\begin{abstract}
Introduction: The purpose of this work is the continuation rate of intra uterine device (IUD) copper in a nulliparous population.
\end{abstract}

Material and method: prospective study in a family planning centre included nulliparous patients between January and December 2012.

Results: A total of 83 nulliparous patients have IUD copper insertion and 11 patients were excluded because of lost follow-up. Finally, 72 nulliparous patients were included in the study. Continuation rate after one-year follow-up was 90,3\% (65/72). Satisfaction rate of patient was high $(93,8 \%)$. Menstruation amount was increased for $84 \%(55 / 65)$ patients, but $75 \%(41 / 55)$ did not show any inconvenient about this. Dysmenorrhea were increased for $80 \%(52 / 65)$ patients, but $80 \%(52 / 65)$ did not show any inconvenient about this.

Conclusion: Continuation rate of IUD copper was high (90\%) after one-year follow-up in nulliparous women. Tolerance was also good for evaluated patients.

Keywords : intra uterine device ; nulliparous 


\section{Introduction}

La France dispose d'une bonne couverture contraceptive. En effet, selon l'Organisation Mondiale de la santé, $75 \%$ des femmes en âge de procréer déclarent prendre un moyen de contraception [1]. Le modèle contraceptif français ou «norme contraceptive » repose sur une utilisation préférentielle de la pilule (50,6\% des françaises) surtout chez les 20-24 ans avec 63,3\% d'utilisation dans cette tranche d'âge [2, 3]. Le dispositif intra-utérin (DIU) ou stérilet vient en deuxième position en fréquence d'utilisation avec $20,7 \%$ d'utilisatrices. Il est principalement proposé aux femmes de plus de 35 ans. Quant au préservatif, il est surtout utilisé au début de la vie sexuelle. Pourtant, l'indice de Pearl du préservatif est peu satisfaisant (>15), particulièrement dans les populations adolescentes [2, 3].

Cette bonne couverture contraceptive française a permis de diminuer le nombre de grossesses non programmées de $50 \%$ à $30 \%$ en une trentaine d'année. Cependant, cette diminution s'est accompagnée d'une augmentation du recours à l'interruption volontaire de grossesse (IVG) en cas de grossesse inattendue, rendant le nombre d'IVG stable autour de 220000 par an depuis sa légalisation en France. En cas d'IVG, deux tiers des patientes indiquent prendre un moyen de contraception et parmi ces patientes, environ $60 \%$ déclarent utiliser la pilule [4-6].

Pour améliorer l'efficacité de la couverture contraceptive française, l'Inspection Général de la Santé (IGAS) propose dans son rapport de 2009 de promouvoir les moyens de contraception réversible de longue durée d'action comme le DIU. Dans les recommandations de la Haute Autorité de Santé (HAS), le DIU au cuivre est une contraception de première intention, y compris pour les patientes nullipares [7]. Pourtant, la prescription du DIU reste marginale chez les nullipares (1,3\%) en France, même après les tempêtes médiatiques de 2012 sur les pilules de $3^{\text {ème }}$ ou $4^{\text {ème }}$ générations $[1,2]$. Des freins à la fois médicaux et sociétaux persistent dans l'utilisation du DIU chez les nullipares. Par exemple, reste encore dans les mémoires le scandale américain des années 70 lié au DIU Dalkon Shield®, DIU en plastique, dépourvu de cuivre, avec un fil tressé, et responsable de nombreux cas de maladie inflammatoire pelvienne (MIP). Pourtant, depuis, de nombreuses publications réalisées sur des cohortes conséquentes, montrent l'absence de sur-risque de MIP ou d'infertilité secondaire liées à l'utilisation du DIU au 
cuivre [8-12]. En France, le DIU est perçu dans la population générale ou médicale comme non indiqué chez la nullipare du fait de problèmes de tolérance [2].

L'objectif principal de notre étude prospective est d'évaluer le taux de continuation à 1 an du DIU au cuivre posé chez des patientes nullipares dans un centre de planification familiale en France. L'objectif secondaire était l'évaluation de la tolérance du DIU au cuivre chez ces patientes nullipares. 


\section{Matériel et Méthodes}

\section{Design de l'étude}

Il s'agit d'une étude prospective unicentrique de cohorte chez des patientes nullipares ayant bénéficié de la pose d'un DIU au cuivre au Planning Familial 35 de Rennes entre le $1^{\text {er }}$ janvier 2012 et le 31 décembre 2012. Dans ce centre de planification en 2012, 2544 consultations médicales ont été réalisées : 325 consultations pré-IVG, 1704 consultations de contraception et 515 consultations de gynécologie (autre que pour la contraception).

Les critères d'inclusion étaient toutes les patientes de plus de 18 ans nullipares demandant une contraception par un DIU au cuivre. Les critères d'exclusion étaient un âge inférieur à 18 ans, une parité supérieure ou égale à 1, le post-abortum immédiat (i.e. IVG de moins de 3 mois), une contre-indication au DIU (des métrorragies inexpliquées, une infection pelvienne en cours, une grossesse en cours...), l'absence de suivi à 1 an.

\section{Patientes et technique de pose du DIU}

Les patientes nullipares vues en consultation de contraception et demandeuses de la pose d'un DIU au cuivre se voyaient proposer de participer l'étude.

Avant la pose du DIU, un prélèvement vaginal à la recherche de Chlamydia trachomatis et de Neisseriae gonorrhoe par PCR a été réalisé. En cas de positivité pour l'un ou l'autre des germes, un traitement antibiotique adapté était réalisé et un nouveau prélèvement vaginal réalisé pour s'assurer de l'efficacité du traitement et donc de l'absence de persistance du germe avant la pose du DIU. En cas de retard de règles, un test urinaire de grossesse était réalisé pour s'assurer de l'absence de grossesse.

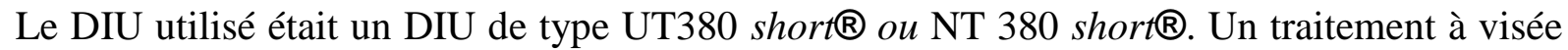
antalgique était prescrit aux patientes à raison de $800 \mathrm{mg}$ d'ibuprofène une heure avant la pose. Une anesthésie locale intra-cervicale à raison de $20 \mathrm{mg}$ de lidocaïne injectés pour moitié à 3 heures et pour moitié à 9 heures a été réalisée aux patientes qui le souhaitaient au moment de la pose du DIU. La pose du DIU s'est déroulée pendant ou en dehors des règles après une antiseptie du vagin à la Bétadine® gynécologique et la réalisation d'une hystérométrie.

\section{Suivi des patientes}

La durée de suivi des patientes était de 12 mois avec 3 consultations proposées à la patiente. Lors de la première consultation, la patiente bénéficiait de la pose du DIU et devait répondre au premier questionnaire. Celui-ci portait sur les caractéristiques socio-démographiques de la patiente (âge, niveau d'étude, activité professionnelle, mutuelle ou CMU, statut marital), les 
antécédents médicaux, chirurgicaux et gynéco-obstétricaux, les facteurs de risque d'infection sexuellement transmissible (utilisation de préservatif, nombre de partenaires sexuels par an, partenaire stable...), les facteurs ayant amenés la patiente à choisir un DIU (sources d'information, inconvénients de la contraception actuelle, bénéfices attendus du DIU...) et les caractéristiques du cycle de la patiente en l'absence de contraception hormonale (dysménorrhée, régularité du cycle, abondance des règles...). Nous avons classé en trois groupes les patientes en fonction de l'abondance des règles : les règles étaient dites de faible abondance lorsque le flux menstruel étaient inférieur à $30 \mathrm{~mL} /$ jour, d'abondance moyenne entrede 30 et $50 \mathrm{~mL} / \mathrm{J}$ et de forte abondance si supérieur $50 \mathrm{~mL} /$ jour. La quantité du flux était calculée en fonction du nombre de protection utilisées par jour et de leur taille (un tampon classé « light » contient $5 \mathrm{~mL}$, classé « régulier » 9 à $12 \mathrm{~mL}$, classé «super » 12 à $15 \mathrm{~mL}$, et classé « super plus » 15 à $18 \mathrm{~mL})$.

Les dysménorrhées ont été classées en dysménorrhées nulles (aucune douleur pendant les règles), faibles (douleurs décrites sans prise d'antalgique ou alors prise de paracetamol ou de phloroglucinol (Spasfon ${ }^{\circledR}$ ) pendant moins de 3 jours), moyennes (si prise d'AINS ou si prise de paracétamol ou de phloroglucinol pendant plus de 3 jours), fortes (si prise d'antalgique de niveau 2 ou si prise d'antalgique de niveau 1 pendant plus de 5 jours).

Lors de la deuxième consultation à 1 mois de la pose du DIU, la tolérance du DIU (douleurs, méno-métrorragies...) et des signes d'infection pelviennes étaient recherchés. La position du DIU par examen clinique (position des fils) était vérifiée. Un deuxième questionnaire était remis à la patiente et portait sur la douleur ressentie lors de la pose du DIU 1 mois avant et évaluée par une échelle visuelle analogique (EVA) de 1 à 10, la durée de la douleur pelvienne ressentie en nombre de jour après la pose, la durée des métrorragies après la pose du DIU (en jour). Des questions portaient également sur l'abondance des règles (quantifiée comme précédemment), sur la durée des règles, sur l'évolution des règles (considérées comme plus abondantes, moins abondantes ou identiques aux règles avant la pose du DIU) et sur les dysménorrhées (classées comme précédemment et aussi selon leur évolution: plus douloureuses, aussi douloureuses ou moins douloureuses qu'avant la pose du DIU). Enfin une partie du questionnaire portait sur la satisfaction des patientes vis-à-vis du DIU évaluée par une EVA de 1 à 10 (1 pas satisfaite et 10 très satisfaite) et sur leur qualité de vie classée en 3 catégories (amélioration, identique et dégradation).

Lors de la $3^{\text {ème }}$ consultation à 1 an, la poursuite ou non de la contraception par DIU, la tolérance du DIU (douleurs, méno-métrorragies...) et des signes d'infection pelviennes étaient recherchés. La position du DIU par examen clinique (position des fils) était vérifiée. 
Un deuxième questionnaire était alors remis à la patiente. Si le DIU était abandonné, les raisons de l'abandon de ce moyen de contraception étaient recherchées. Si le DIU était maintenu, des questions portaient également sur l'abondance des règles (quantifiée comme précédemment), sur la durée des règles, sur l'évolution des règles (considérées comme plus abondantes, moins abondantes ou identiques aux règles avant la pose du DIU) et sur les dysménorrhées (classées comme précédemment et aussi selon leur évolution: plus douloureuses, aussi douloureuses ou moins douloureuses qu'avant la pose du DIU). Une partie du questionnaire portait sur la satisfaction des patientes vis-à-vis du DIU évaluée par une EVA de 1 à 10 (1 pas satisfaite et 10 très satisfaite) et sur leur qualité de vie classée en 3 catégories (amélioration, identique et dégradation). De même le questionnaire EVAPIL est remis à la patiente si elle avait gardé son moyen de contraception par DIU au cuivre 12 mois. Ce questionnaire EVAPIL est décrit précédemment [13]. Brièvement, les 14 symptômes suivants sont recherchés : nervosité/irritabilité, nausées, maux de tête/migraines, abondance des règles, peau grasse, acné, prise de poids, sensation de jambes lourdes, mastodynies, dysménorrhées, ballonements, spottings, régularité du cycle, plaisir lors des rapports sexuels. Ces symptômes permettent d'explorer 7 aspects : le bien-être général, l'état psychologique, les désordres intestinaux, les désordres cutanés, les seins, le cycle et la sexualité. Ces symptômes sont évalués de façon à savoir s'ils sont ou non plus présents qu'avant et de mesurer la gêne occasionnée, graduée de 1 (absence de gêne) à 4 (gêne importante). Si la patiente n'est pas revenue à sa consultation de suivi à un an de la pose du DIU, la patiente est recontactée par téléphone ou par courrier. Si la patiente ne peut pas venir à sa consultation (pour cause de déménagement ou autre), les questionnaires sont remplis par téléphone ou par mail à sa convenance.

\section{Critères de jugement}

Le critère de jugement principal était le taux de continuation à un an du DIU au cuivre par la patiente. Le critère de jugement secondaire était la satisfaction des patientes mesurée par l'EVA (de 0 à 10) et par le score moyen EVAPIL calculé pour chaque patiente [13]. A chaque symptôme a été attribué un score selon son degré d'intensité et de fréquence (A). Ce score a ensuite été multiplié par le degré d'inconfort de la patiente (gêne allant de 1 à 4) (B). Le produit $(\mathrm{AxB}=\mathrm{C})$ a été pondéré selon le nombre de réponse possible à l'item $(\mathrm{D})$. Le score total $(\mathrm{CxD}=\mathrm{T})$ a été rapporté au nombre de scores valides (i.e. non nuls) (N) pour obtenir le score moyen $(\mathrm{T} / \mathrm{N})$. L'étude de Graesslin et col a montré que le score était corrélé à la satisfaction des patientes : plus le score est proche de 0 et plus la patiente est satisfaite de sa 
contraception [13]. Le score maximum est de 12. Il est à noter qu'un score de 0 pour un item ne signifie pas que le symptôme n'est pas présent mais qu'il n'occasionne aucun inconfort.

Analyse Statistique

Les tests statistiques utilisés ont été le test du Chi2 ou le test exact de Fisher, le cas échéant, pour les variables qualitatives et le test $t$ de Student pour les variables continues. Une valeur du p inférieure ou égale à 0,05 a été retenue comme statistiquement significative. Les analyses statistiques ont été réalisées à l'aide du logiciel SAS ${ }^{\circledR} 9.1$ (SAS Institute, Cary, NC, ÉtatsUnis). 


\section{Résultats}

\section{Caractéristiques de la population étudiée}

Un total de 96 patientes nullipares a été inclus dans l'étude, 11 patientes ont été exclues car perdues de vue au décours de la pose du DIU au cuivre et 13 autres patientes ont également été exclues du fait de la pose d'un DIU au levonorgestrel (Miréna (B). Au total, 72 patientes ont participé à l'étude (figure 1).

Figure 1 : Diagramme d'Inclusion dans l'Etude (DIU : Dispositif Intra Utérin)

Figure 1 : Flowchart of study recruitment

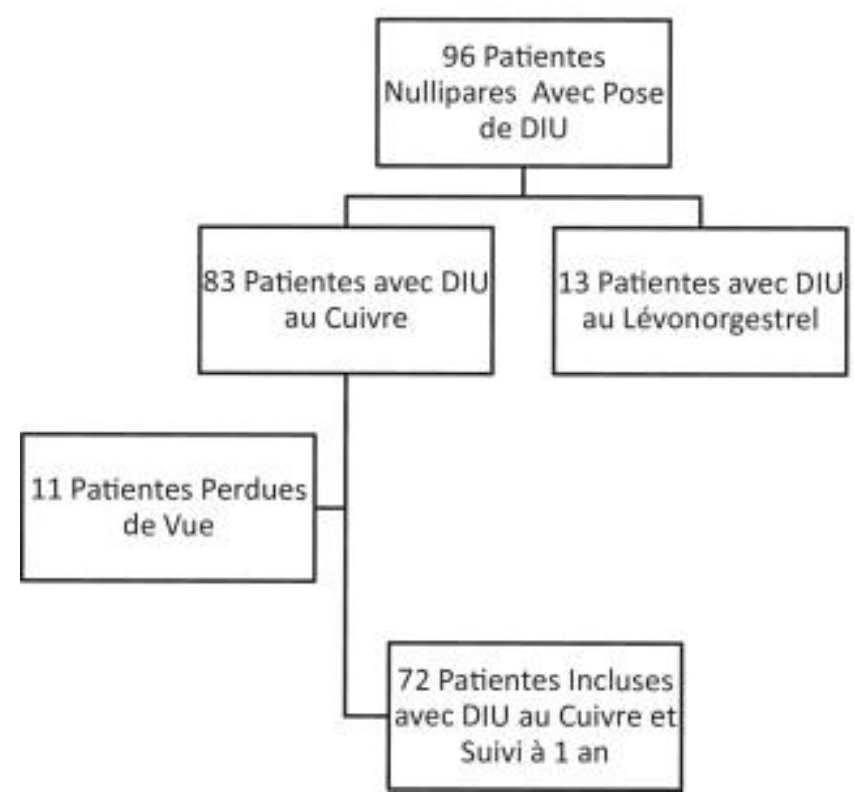

La moyenne d'âge des patientes était de 26 ans $( \pm 4,5)$ ans. Les caractéristiques de la population étudiées sont décrites dans le tableau 1. Six patientes présentent une contreindication à la pilule oestro-progestative : 3 patientes ont des migraines sévères (avec aura pour l'une d'entre elle), une patiente présente un déficit en anti-thrombine III, une patiente présente une recto-colique hémorragique et une patiente présente un trouble psychiatrique bipolaire traitée par quétiapine (Xéroquel®). La contraception utilisée avant la pose du DIU était une contaception oestro-progestative pour 52\% d'entre elles et le préservatif pour 36\% d'entre elles. Les motifs principaux indiqués par les patientes pour changer de contraception vers un DIU était le manque d'efficacité des contraceptions utilisées jusqu'alors pour 24\% 
d'entre-elles, l'observance (oubli de pilule) pour 26\% d'entre elles ou encore les effets secondaires hormonaux (de type troubles de la libido, sécheresse vaginale, migraines, mastodynies....) pour 37\% d'entre elles. Les principaux bénéfices attendus du DIU par les patientes étaient l'absence d'hormone pour $62 \%$ d'entre elles, sa facilité d'utilisation pour $33 \%$ d'entre elles et son efficacité pour $24 \%$ d'entre elles.

\section{Pose du DIU au cuivre et contrôle à un mois}

Un prélèvement vaginal a été réalisé chez toutes les patientes, excepté chez deux patientes de plus de 25 ans avec un partenaire stable. Deux infections ont été dépistées : une patiente avec un portage du Chlamydia Trachomatis et une patiente avec du Neisseria Gonorrhoeae. Ces patientes ont été traitées et un nouveau prélèvement a montré l'absence de persistance du germe avant la pose $\mathrm{du}$ DIU. Soixante-douze DIU au cuivre ont été posés dont 65 UT380 short®, 4 NT 380 short®, 2 Cu375 SL® et 1 UT 380 standard®. De l'ibuprofène a été donné juste avant la pose du DIU pour 64/72 (89\%) des patientes. Une anesthésie locale intra-cervicale a été réalisée au moment de la pose du DIU pour $6(8 \%)$ patientes (à la demande de celle-ci). Aucun échec de pose n'a été enregistré. Cette pose s'est déroulée pendant les règles pour $65 \%$ des patientes et en dehors des règles pour $35 \%$ d'entre elles. La douleur moyenne maximale ressentie par les patientes au moment de la pose était de $6,7( \pm 2,0)$ sur l'EVA. Il n'est pas enregistré de différence significative sur la douleur entre une pose de DIU pendant les règles $(6,6( \pm 2,0)$ et une pose en dehors des règles $(6,7( \pm 2,1))(\mathrm{p}=0,75)$. La pose du DIU précédée d'une anesthésie locale intra-cervicale fait significativement baisser l'EVA lors de la pose de 6,9 $( \pm 1,9)$ à $4,3( \pm 2,7)(\mathrm{p}=0,003)$. Treize patientes $(18 \%)$ ont présenté un malaise vagal lors de la pose. Ces malaises ont été de résolution rapide et spontanée.

Le suivi à un mois de la pose du DIU au cuivre est décrit tableau 2. Ce suivi ne concerne que les 57 patientes qui ont réalisé leur consultation de contrôle au centre de planification et ont rempli le questionnaire d'auto-évaluation. Aucune infection génitale haute n'a été repérée lors de cette consultation de contrôle. Deux patientes ont consulté en urgence. La première patiente a consulté 7 jours après la pose pour des douleurs et des métrorragies. Comme les explorations cliniques et paracliniques n'ont trouvé aucune anomalie, le DIU a été laissé en place. La seconde patiente a consulté en urgence 20 jours après la pose pour l'apparition de douleur et de métrorragies. Les explorations cliniques et paracliniques n'ont rien retrouvé et le DIU a été laissé en place. Cette $2^{\text {ème }}$ patiente a consulté de nouveau 7 jours après pour les 
mêmes symptômes, motivant le retrait du DIU. La satisfaction des patientes évaluées par l'EVA à un mois de la pose du DIU est de $8,2( \pm 1,5)$.

\section{Tolérance et efficacité de la contraception par DIU au cuivre à 1 an de la pose}

Le taux de continuation de la contraception par DIU au cuivre à 1 an (critère de jugement) dans notre population de patientes nullipares incluses étaient de 90,3\% (65/72). Si les 11 patientes perdues de vue sont inclues dans la population d'étude, ce taux de continuation est à 78,3\% (65/83). Sept patientes ont dû retirer leur DIU au cuivre : 5 patientes pour des douleurs pelviennes (cycliques ou non, avec des dyspareunies ou non), 1 patiente pour des métrorragies et 1 patiente pour la survenue d'une grossesse. La patiente avec une grossesse malgré le DIU au cuivre a bénéficié d'une interruption volontaire de grossesse et ensuite souhaité reprendre une contraception par DIU au cuivre. Deux patientes ont fait retirer leur DIU moins de 6 mois après la pose et 5 patientes entre 6 et 12 mois après la pose du DIU.

Dans le suivi, une infection génitale a été diagnostiquée : une patiente a consulté à 5 mois de la pose du DIU pour des métrorragies brunâtres et des douleurs pelviennes. Le prélèvement vaginal a montré une infection à chlamydia trachomatis, les examens paracliniques montraient l'absence de syndrome inflammatoire biologique et une imagerie génitale normale à l'échographie. La patiente a reçu un traitement monodose par azithromycine et le DIU a été laissé en place. Le contrôle par prélèvement vaginal est revenu négatif pour le chlamydia trachomatis à 6 mois et la symptomatologie s'est complètement amendée.

Le questionnaire de tolérance a pu être rempli par les 65 patientes qui ont poursuivi leur contraception par DIU à 1 an de la pose. Les réponses à ce questionnaire sont présentées dans le tableau $n^{\circ} 3$. La tolérance a également été évaluée par le score EVAPIL qui reporte les symptômes décrits par la patiente et qui pondère ces symptômes en fonction de la gêne occasionnée par ce symptôme. Le score moyen EVAPIL pour notre population est de 1,06 $( \pm 0,84)$. Le tableau 4 montre le détail des réponses au questionnaire EVAPIL dans la population d'étude à un an de la pose du DIU au cuivre. 


\section{Discussion}

Notre étude montre un taux de continuation à 1 an du DIU au cuivre à 90,3\% chez une population de patientes nullipares françaises. La satisfaction des patientes vis-à-vis de cette contraception est également élevée avec un taux de patientes satisfaites à 93,8\% chez les patientes continuant cette contraception plus de 1 an. Avec le DIU au cuivre, selon les réponses à l'échelle EVAPIL, une augmentation de l'abondance des règles est observée chez 84,6\% (55/65) des patientes dont 75\% (41/55) se disent peu ou pas gênées par ce symptôme. De même, une augmentation des dysménorrhées est observée chez 80\% (52/65) des patientes dont 58\% (30/52) se disent peu ou pas gênées par ce symptôme. A notre connaissance, il s'agit de la première étude sur le taux de continuation de la contraception par DIU au cuivre chez des patientes nullipares en France.

Par rapport à la littérature internationale, nous observons un taux de continuation comparable $\mathrm{du}$ DIU au cuivre chez les nullipares. Ainsi Otero-Flores et al retrouvent un taux de continuation de $85 \%$ à 1 an chez 780 femmes mexicaines nullipares [14]. De même, plusieurs études retrouvent des taux de continuation élevées et similaires entre les populations de patientes nullipares et multipares [15-19]. Dans l'étude COCON, le taux de continuation du DIU quelque soit sa nature est de $85 \%$ dans la population générale. Ce taux de continuation est supérieur à celui enregistré par la contraception oestro-progestative orale avec des taux de continuation à 1 an autour de 68\% [20]. Les motifs d'arrêt de la contraception par DIU au cuivre sont une mauvaise tolérance liée à deux maîtres symptômes : les dysménorrhées et les ménorragies. Dans notre étude, 7 retraits de DIU ont été nécessaires devant des saignements dans 2 cas et des douleurs dans 4 cas, parfois ces deux symptômes sont associés. Ces motifs d'arrêt sont retrouvés dans la littérature et les femmes nullipares seraient légèrement plus susceptibles d'arrêter leur contraception par DIU au cuivre pour des méno-métrorragies et/ou des dysménorrhées que les patientes multipares [15, 21, 22]. Une bonne information préalable des patientes sur ces différents symptômes permettrait de diminuer le taux de discontinuation de la contraception par DIU au cuivre [23].

Une des réticences à l'utilisation du DIU au cuivre chez les patientes nullipares est la crainte des difficultés liées à la pose. Dans les études portant sur des grands effectifs de patientes, il est enregistré environ 1,2\% de difficultés liées à la pose, difficultés représentées par des douleurs importantes et/ou des saignements. Ces difficultés sont significativement liées à la fois à la nulliparité et à l'expérience du médecin réalisant la pose [21, 23, 24]. Dans notre série, il a toujours été possible de réaliser la pose du DIU. Dix-huit pourcents (13/72) des patientes ont présenté un malaise vagal, de résolution rapide et spontanée. L'EVA moyenne 
lors de la pose reste élevée chez les patientes $(6,3 \pm 2,4)$, mais sur une courte durée, malgré la prise d'ibuprofène systématique. Il n'est pas retrouvé de différence significative sur les douleurs en fonction du moment de la pose pendant les règles ou en dehors des règles. Ces données sont confirmées par la littérature : aucune étude n'a permis de mettre en évidence un bénéfice sur les douleurs à la prise d'ibuprofène avant la pose du DIU [24, 25]. De même, aucune étude ne montre une diminution des douleurs lors de la pose d'un DIU pendant les règles par rapport à une pose en dehors des règles $[24,26]$. La prise de misoprostol a été évaluée pour diminuer la douleur de la pose d'un DIU mais n'a pas montré de bénéfice, au prix d'augmentation des effets indésirables comme les nausées ou les crampes intestinales [27-29]. Enfin, une anesthésie para-cervicale a également été proposée mais sans différence significative sur les douleurs [30]. Cependant, dans notre étude la réalisation d'une anesthésie intra-cervicale au moment de la pose du DIU permet de diminuer significativement ces douleurs. Ce bénéfice de l'anesthésie intra-cervicale est probablement à réévaluer dans une étude dédiée à montrer cet objectif. Ces résultats (absence de bénéfice sur les douleurs de la pose d'un DIU pendant les règles et le potentiel bénéfice antalgique d'une anesthésie intracervicale) doivent permettre de faciliter l'accès au DIU à la population générale en s'affranchissant des contraintes logistiques de pose nécessitant de coordonner planning de consultation du médecin et menstruations de la patiente et en diminuant la douleur certes fugace mais élevée de la pose du DIU.

Notre étude souffre d'un certain nombre de limites. Tout d'abord, nous avons 11 patientes perdues de vue et certaines d'entre elles ont pu faire retirer leur DIU dans un autre centre. Ces perdues de vues peuvent faire varier notre taux de continuation à 1 an entre 90,3\% (65/72) et 78,3\% (65/72+11). Ensuite, il s'agit d'une étude concernant les patientes d'un centre de planification familiale avec un probable biais de sélection: les patientes recrutées sont particulièrement motivées par la pose d'un DIU. En effet, un certain nombre de ces patientes présente des contre-indications à l'utilisation de la contraception oestro-progestative, d'autres patientes sont à la recherche d'une contraception efficace non hormonale et avaient consulté plusieurs médecins avant de trouver le centre de planification acceptant de réaliser la pose d'un DIU chez une patiente nullipare. Ensuite, comme le montrent Suhonen et al, le bon «counselling »sur les symptômes potentiels du DIU apporté par une équipe de planification influe favorablement sur le taux de continuation du DIU [23]. Ces éléments font que le taux de continuation du DIU au cuivre dans notre population est particulièrement élevé, à 90,3\%, supérieur à ce qui est retrouvé dans la littérature dont le taux de continuation oscille entre $78 \%$ et $87 \%$ [31]. Concernant l'évaluation des ménorragies, les auteurs ont fait le choix de ne 
pas utiliser l'échelle de Higham. En effet, un certain nombre de patientes utilisaient des coupes menstruelles rendant impossible l'utilisation de ce score pour celles-ci. Par ailleurs, certaines interrogations existent sur ce score aux yeux des auteurs : un score de Higham de 100 points définit une ménorragie $(>80 \mathrm{ml}$ selon le Collège National des Gynécologues Obstétriciens Français), soit 5 tampons ou serviettes pleines. Or toutes les femmes utilisent au moins 5 protections complètes pendant la totalité de leur règles, ce qui classeraient toutes les patientes femmes ménorragiques selon ce score. Par ailleurs, nous avons utilisé l'échelle EVAPIL pour évaluer la tolérance et la satisfaction des patientes quant à l'utilisation du DIU. Quoique cette échelle ait été conçue pour évaluer la contraception orale, nous pouvons montrer un score moyen EVAPIL de satisfaction à $1,06( \pm 0,84)$ dans notre population porteuse d'un DIU au cuivre comparable au score moyen de $0,68( \pm 0,74)$ des patientes satisfaites de leur contraception orale dans l'étude de Graesslin et al [13]. Les auteurs reconnaissent que l'utilisation de l'échelle EVAPIL-R évaluant tous les moyens de contraception aurait été plus pertinente, mais cette échelle n'était pas encore disponible au moment de la présente étude [32]. Enfin, le fait de faire remplir de nombreux questionnaires et d'entrer dans une étude introduit probablement un biais d'observation, ce qui pose le problème de la transposabilité des résultats obtenus à la population générale. Cependant, notre étude observationnelle est réalisée dans un centre de planification ancrée dans une activité quotidienne de prise en charge des patientes non sélectionnées, rendant des résultats sur l'acceptabilité du DIU dans une population de patientes nullipares tout à fait pertinents.

En conclusion, notre étude permet de montrer un taux de continuation à 1 an du DIU au cuivre à 90,3\% dans une population de patiente nullipare, avec un excellent taux de satisfaction et une excellente tolérance. Ce moyen de contraception de longue durée d'action mérite d'être plus largement diffusé auprès de la population française nullipare pour satisfaire la demande croissante des patientes pour ce type de contraception. Sa large diffusion permettrait probablement de diminuer le nombre de grossesses non programmées, tout au moins pour les patientes qui en bénéficieraient. 


\section{Références}

1. United Nations Fertility and Family Planning Section Model-based estimates: countries contraceptive prevalence. Percentage of married or in-union women aged 15 to 49 who are currently using any method of contraception. http://www.un.org/esa/population/publications/WCU2012/MainFrame.html, 2012.

2. Bajos, N., et al., Enquête Fécond. La contraception en France: nouveau contexte, nouvelles pratiques ? Populations et Sociétés. , 2012. 492(Sep).

3. Haute Autorité de Santé. Etats des lieux des pratiques contraceptives et des freins à l'accès et au choix d'une contraception adaptée. . 2013.

4. Lavoue, V., et al., [Medical abortion from 12 through 14 weeks' gestation: a retrospective study with 126 patients]. J Gynecol Obstet Biol Reprod (Paris), 2011. 40(7): p. 626-32.

5. Bajos, N., et al., Pourquoi le nombre d'avortements n'a-t-il pas baissé en France depuis 30 ans ? Populations et Sociétés., 2004. 407(Dec).

6. Lavoue, V., et al., Screening for Chlamydia trachomatis using self-collected vaginal swabs at a public pregnancy termination clinic in France: results of a screen-andtreat policy. Sex Transm Dis, 2012. 39(8): p. 622-7.

7. ANAES, Stratégies de choix des méthodes contraceptives chez la femme. Recommandations pour la pratique clinique. . 2004.

8. Darney, P.D., Time to pardon the IUD? N Engl J Med, 2001. 345(8): p. 608-10.

9. Grimes, D.A., Intrauterine device and upper-genital-tract infection. Lancet, 2000. 356(9234): p. 1013-9.

10. Grimes, D.A., Intrauterine devices and infertility: sifting through the evidence. Lancet, 2001. 358(9275): p. 6-7.

11. Farley, T.M., et al., Intrauterine devices and pelvic inflammatory disease: an international perspective. Lancet, 1992. 339(8796): p. 785-8.

12. Hubacher, D., et al., Use of copper intrauterine devices and the risk of tubal infertility among nulligravid women. N Engl J Med, 2001. 345(8): p. 561-7.

13. Graesslin, 0., et al., The EVAPIL scale, a new tool to assess tolerance of oral contraceptives. Contraception, 2009. 80(6): p. 540-54.

14. Otero-Flores, J.B., F.J. Guerrero-Carreno, and L.A. Vazquez-Estrada, A comparative randomized study of three different IUDs in nulliparous Mexican women. Contraception, 2003. 67(4): p. 273-6.

15. Hubacher, D., Copper intrauterine device use by nulliparous women: review of side effects. Contraception, 2007. 75(6 Suppl): p. S8-11.

16. Prager, S. and P.D. Darney, The levonorgestrel intrauterine system in nulliparous women. Contraception, 2007. 75(6 Suppl): p. S12-5.

17. Garbers, S., et al., Continuation of copper-containing intrauterine devices at 6 months. Contraception, 2013. 87(1): p. 101-6.

18. Deans, E.I. and D.A. Grimes, Intrauterine devices for adolescents: a systematic review. Contraception, 2009. 79(6): p. 418-23.

19. Bayer, L.L., et al., Adolescent experience with intrauterine device insertion and use: a retrospective cohort study. Contraception, 2012. 86(5): p. 443-51.

20. Trussell, J. and B. Vaughan, Contraceptive failure, method-related discontinuation and resumption of use: results from the 1995 National Survey of Family Growth. Fam Plann Perspect, 1999. 31(2): p. 64-72, 93. 
21. Harrison-Woolrych, M., J. Ashton, and D. Coulter, Insertion of the Multiload Cu375 intrauterine device; experience in over 16,000 New Zealand women. Contraception, 2002. 66(6): p. 387-91.

22. Trignol-Viguier, N., E. Blin, and H. Marret, [Intra-uterine device and nulliparous women]. Gynecol Obstet Fertil, 2014. 42(6): p. 432-7.

23. Suhonen, S., et al., Clinical performance of a levonorgestrel-releasing intrauterine system and oral contraceptives in young nulliparous women: a comparative study. Contraception, 2004. 69(5): p. 407-12.

24. Hubacher, D., et al., Pain from copper intrauterine device insertion: randomized trial of prophylactic ibuprofen. Am J Obstet Gynecol, 2006. 195(5): p. 1272-7.

25. Chor, J., et al., Ibuprofen prophylaxis for levonorgestrel-releasing intrauterine system insertion: a randomized controlled trial. Contraception, 2012. 85(6): p. 558-62.

26. Whiteman, M.K., et al., When can a woman have an intrauterine device inserted? A systematic review. Contraception, 2013. 87(5): p. 666-73.

27. Espey, E., et al., Misoprostol for intrauterine device insertion in nulliparous women: a randomized controlled trial. Am J Obstet Gynecol, 2014. 210(3): p. 208 e1-5.

28. Edelman, A.B., et al., Effects of prophylactic misoprostol administration prior to intrauterine device insertion in nulliparous women. Contraception, 2011. 84(3): p. 234-9.

29. Lesavre, M., G. Legendre, and H. Fernandez, [Use of misoprostol in gynaecology]. J Gynecol Obstet Biol Reprod (Paris), 2014. 43(2): p. 190-4.

30. Mody, S.K., et al., Pain control for intrauterine device insertion: a randomized trial of 1\% lidocaine paracervical block. Contraception, 2012. 86(6): p. 704-9.

31. Ali, M.M., M.H. Park, and T.D. Ngo, Levels and determinants of switching following intrauterine device discontinuation in 14 developing countries. Contraception, 2014. 90(1): p. 47-53.

32. Clerson, P., et al., EVAPIL-R Scale: Continuous development and validation of a tool to assess patient-reported tolerability of different contraceptive methods in longitudinal studies. Clin Ther, 2014. 36(5): p. 638-647 e3. 
Tableau 1 : Caractéristiques des patientes $(n=72)$

Table 1 : Characteristic of patients $(n=72)$

\begin{tabular}{ll}
\hline Variables & Effectif n \\
\hline Âge (années) & \\
$<20$ ans & $2(3 \%)$ \\
$20-25$ ans & $56(78 \%)$ \\
$26-30$ ans & $8(11 \%)$ \\
$>30$ ans & $6(8 \%)$
\end{tabular}

Statut marital

Célibataire

$15(19 \%)$

En couple (marié, PACS, union libre)

$54(74 \%)$

Non renseigné

$6(7 \%)$

Nombre de partenaire an

Partenaire stable $>1$ an

$56(78 \%)$

$>1$ et $<5$ partenaires par an

$13(18 \%)$

$>5$ partenaires par an

$3(4 \%)$

Couverture sociale

Mutuelle

$66(92 \%)$

CMU complémentaire

$3(4 \%)$

Non renseigné

$3(4 \%)$

Niveau d'étude

Etudes Supérieures

$62(86 \%)$

Niveau Baccalauréat

$3(4 \%)$

Niveau Inférieur au Baccalauréat

$0(0 \%)$

Non renseigné

$7(10 \%)$

Statut professionnel

Etudes (études supérieures, lycée...)

$28(39 \%)$

Travail

$32(44,5 \%)$

Chômage

$11(15,2 \%)$

Femme au foyer

$0(0 \%)$

Non renseigné

$1(1,3 \%)$

Tabagisme

Oui

$35(49 \%)$ 
Non

$37(51 \%)$

Antécédent d'IVG

Oui

$13(18 \%)$

Non

$53(74 \%)$

Non renseigné

$6(8 \%)$

Contre-indication à la pilule oestro-progestative

Oui

$6(8 \%)$

Non

$63(88 \%)$

Non renseigné

$3(4 \%)$

Contraceptions utilisées avant la pose du DIU :

Pilule Oestro-progestative

$37(51 \%)$

Anneau vaginal (Nuvaring®)

$1(1 \%)$

Implant sous-cutané (Nexplanon $®)$

$1(1 \%)$

DIU

$2(3 \%)$

Préservatifs

$26(36 \%)$

Méthodes naturelles

$3(4 \%)$

Non renseigné

$2(3 \%)$

Abondance des règles

Faible

$19(26 \%)$

Moyenne

$37(51 \%)$

Forte

$6(8 \%)$

Dysménorrhées

Nulles

$33(46 \%)$

Faibles

$19(26 \%)$

Moyennes

$13(18 \%)$

Fortes

$1(1 \%)$

DIU : dispositif Intra-Utérin ; 
Tableau 2 : Suivi à 1 mois de la pose du Dispositif Intra-Utérin

Table 2 : One month follow-up after intra uterine device insertion

\begin{tabular}{ll}
\hline Variables & $\begin{array}{l}\text { Moyenne } \\
\text { effectif* }\end{array}$ (écart-type) \\
\hline Durée de la douleur après la pose (jours) & $2,8( \pm 3,5)$ \\
Durée des métrorragies après la pose (jours) & $5,4( \pm 8,7)$ \\
Les règles à 1 mois de la pose du DIU sont & \\
Plus abondantes qu'avant & $31(54 \%)$ \\
Identiques & $15(26,3 \%)$ \\
Moins abondantes qu'avant & $2(3,5 \%)$ \\
NR & $9(16 \%)$ \\
L'abondance des règles sous DIU au cuivre est & \\
Faible & \\
Moyenne & \\
Forte & $24(16 \%)$ \\
& $24(42 \%)$
\end{tabular}

Les dysménorrhées sous DIU au cuivre sont

$\begin{array}{ll}\text { Nulles } & 11(19,3 \%) \\ \text { Faibles } & 20(35,1 \%) \\ \text { Moyennes } & 22(38,6 \%) \\ \text { Fortes } & 4(7 \%) \\ \text { action (EVA) } & 8,2( \pm 1,5) \\ \text { Até de vie avec le DIU est } & \\ \text { Améliorée } & 47(82 \%) \\ \text { Identique } & 9(16 \%) \\ \text { dégradée } & 1(2 \%)\end{array}$

*effectifs de 57 patientes

EVA : échelle visuelle analogique 
Tableau 3 : Suivi à 1 an de la pose du dispositif intra-utérin

Table 3 : One year follow-up after intra uterine device insertion

\begin{tabular}{ll}
\hline Variables & $\begin{array}{l}\text { Moyenne (écart-type) } \\
\text { effectif n }(\%)\end{array}$ \\
\hline Taux de continuation à 1 an & $65 / 72(90,3 \%)$ \\
Durée des règles (jours) & $6,2( \pm 2,1)$ \\
Cycle menstruel régulier & $54 / 65(83,1 \%)$ \\
L'abondance des règles sous DIU au cuivre est* & \\
$\quad$ Faible & $13 / 65(20,0 \%)$ \\
$\quad$ Moyenne & $32 / 65(49,2 \%)$ \\
$\quad$ Forte & $21 / 65(32,3 \%)$ \\
L'abondance des règles par rapport à avant le DIU** : & \\
$\quad$ Augmentation & $22 / 59(37,3 \%)$ \\
Stable & $33 / 59(55,9 \%)$ \\
$\quad$ Diminution & $4 / 59(6,8 \%)$ \\
Dysménorrhées sous DIU au cuivre* & \\
Nulles & $9 / 65(13,8 \%)$ \\
Faibles & $32 / 65(49,2 \%)$ \\
Moyennes & $22 / 65(33,8 \%)$ \\
Fortes & $2 / 65(3,0 \%)$
\end{tabular}

Les dysménorrhées par rapport à avant le DIU*** :

Augmentation

$5 / 31(16,1 \%)$

Stable

$22 / 31(70,1 \%)$

Diminution

$4 / 31(12,9 \%)$

Satisfaction

Satisfaites

$61 / 65(93,8 \%)$

Peu satisfaites

$3 / 65(4,6 \%)$

Insatisfaites

$1 / 65(1,5 \%)$

\footnotetext{
*effectif de 65 patientes qui ont poursuivi leur contraception par DIU 1 an

**effectif de 59 patientes pour qui l'abondance des règles a pu être évaluée avant la pose du DIU parmi les 65 patientes qui ont poursuivi leur contraception par DIU ***effectif de 31 patientes pour qui les dysménorrhées ont pu être évaluée avant la pose du DIU parmi les 65 patientes qui ont poursuivi leur contraception par DIU
} 
Tableau 4 : Description des réponses au questionnaire EVAPIL dans la population d'étude.

Table 4 : EVAPIL responses in study population

\begin{tabular}{|c|c|c|c|c|c|}
\hline & \multicolumn{5}{|c|}{ DIU cuivre (n=65) } \\
\hline & \multirow{2}{*}{ Total } & \multicolumn{4}{|c|}{ Gêne } \\
\hline & & 1 & 2 & 3 & 4 \\
\hline \multicolumn{6}{|c|}{ Irritabilité/Nervosité } \\
\hline un peu plus & 13 & 4 & 8 & 1 & \\
\hline beaucoup plus & 0 & & & & \\
\hline Total & $20,0 \%$ & & & & \\
\hline \multicolumn{6}{|l|}{ Nausées } \\
\hline un peu plus & 4 & 1 & 2 & 1 & \\
\hline beaucoup plus & 2 & & & 2 & \\
\hline Total & $9,2 \%$ & & & & \\
\hline \multicolumn{6}{|c|}{ Maux de tête/Migraines } \\
\hline un peu plus & 5 & 1 & 3 & 1 & \\
\hline beaucoup plus & 1 & & & & 1 \\
\hline Total & $9,2 \%$ & & & & \\
\hline \multicolumn{6}{|l|}{ Abondance } \\
\hline un peu plus & 20 & 13 & 5 & 2 & \\
\hline plus & 17 & 1 & 13 & 3 & \\
\hline beaucoup plus & 18 & 1 & 8 & 4 & 5 \\
\hline Total & $84,6 \%$ & & & & \\
\hline \multicolumn{6}{|l|}{ Peau grasse } \\
\hline un peu plus & 17 & 11 & 6 & & \\
\hline plus & 0 & & & & \\
\hline beaucoup plus & 1 & & & & 1 \\
\hline Total & $27,7 \%$ & & & & \\
\hline \multicolumn{6}{|l|}{ Acné } \\
\hline un peu plus & 13 & 7 & 6 & & \\
\hline plus & 0 & & & & \\
\hline beaucoup plus & 1 & & & & 1 \\
\hline Total & $21,5 \%$ & & & & \\
\hline \multicolumn{6}{|l|}{ Prise de poids } \\
\hline$<2 \mathrm{~kg}$ & 2 & 2 & & & \\
\hline $2>x<5 k g$ & 6 & 1 & 3 & 1 & 1 \\
\hline$>5 \mathrm{~kg}$ & 1 & & & 1 & \\
\hline Total & $13,8 \%$ & & & & \\
\hline \multicolumn{6}{|l|}{ Jambes lourdes } \\
\hline un peu plus & 5 & 3 & 2 & & \\
\hline plus & 0 & & & & \\
\hline beaucoup plus & 3 & & & 2 & 1 \\
\hline Total & $12,3 \%$ & & & & \\
\hline \multicolumn{6}{|l|}{ Mastodynies } \\
\hline un peu plus & 16 & 9 & 7 & & \\
\hline plus & 8 & & 7 & 1 & \\
\hline beaucoup plus & 3 & & 1 & 1 & 1 \\
\hline Total & $41,5 \%$ & & & & \\
\hline Dysménorrhées & & & & & \\
\hline
\end{tabular}




\begin{tabular}{|l|c|c|c|c|c|}
\hline un peu plus & 30 & 9 & 19 & 2 & \\
\hline beaucoup plus & 22 & & 2 & 10 & 10 \\
\hline Total & $80,0 \%$ & & & & \\
\hline Ballonnements & & & & & \\
\hline un peu plus & 9 & 3 & 6 & & \\
\hline beaucoup plus & 2 & & & 2 & \\
\hline Total & $16,9 \%$ & & & & \\
\hline Spottings & & & & & \\
\hline quelques fois/1 protection & 13 & 10 & 2 & 1 & \\
\hline quelques fois/plusieurs protections & 1 & & 1 & & \\
\hline souvent /1 protection & 5 & & 1 & 4 & \\
\hline souvent /plusieurs protections & 4 & & & 3 & 1 \\
\hline Total & $35,3 \%$ & & & & \\
\hline Absence mensuelle de règles & & & & & \\
\hline Parfois & 3 & & 2 & 1 & \\
\hline Souvent & 0 & & & & \\
\hline La plus part du temps & 0 & & & & \\
\hline Jamais & 0 & & & & \\
\hline Total & $4,6 \%$ & & & & \\
\hline Appréciation des rapports sexuels & & & & & \\
\hline un peu moins bien & 4 & & 3 & 1 & \\
\hline beaucoup moins bien & 0 & & & & \\
\hline Plus du tout & 1 & & & & 1 \\
\hline Total & & & & & \\
\hline
\end{tabular}

